\title{
Von der Irritation zur Reflexivität - Zum Potenzial fachdidak- tischer Exkursionen für die Professionalisierung angehender Geographielehrkräfte
}

\footnotetext{
*segbers@geo.uni-frankfurt.de, Institut für Humangeographie, Fachbereich Geowissenschaften/Geographie, Goethe-Universität Frankfurt a.M.

*** eberth@idn.uni-hannover.de, Institut für Didaktik der Naturwissenschaften (Didaktik der Geographie), Leibniz Universität Hannover
}

eingereicht am 21.07.2016; akzeptiert am 13.01.2017

\begin{abstract}
Der Beitrag zeigt anhand exemplarischer Situationen einer geographischen Exkursion nach Kenia die Bedeutung der methodischen Großform Exkursion für die geographische Bildung und die Professionalisierung angehender Geographielehrkräfte. Durch teilnehmende Beobachtung, Tagebuchanalyse und Interviews wurden die Wahrnehmungen der Teilnehmenden zu erfassen versucht. Die Ergebnisse zeigen das Potenzial der Irritation, die Reflexionsprozesse anregt, die zur (Selbst-)Reflexivität führen können und dadurch einen Beitrag zur Inter- und Transkulturellen Kompetenz zu leisten vermögen.
\end{abstract}

Keywords: Afrikabilder, Erfahrung, Exkursion, Professionalisierung von Lehrkräften, Reflexivität

\section{About irritation and reflection - The potential of didactic excursions regarding the professionalisation in geographic teachers training}

\begin{abstract}
The paper makes use of typical situations from a geography field study in Kenya to show the importance of methodical large-scale field trips for geographical education and the professional development of future geography teachers. Attempts were made to record the perceptions of participants using observation, journal analysis and interviews. The results display the potential behind initial confusion that can lead to reflection processes, can lead to (self-)reflexivity and thereby be capable of making a contribution to both intercultural and transcultural competencies.
\end{abstract}

Keywords: images of africa, experience, field trip, geographical teacher education, reflexivity

\section{$1 \quad$ Eine Reise nach Afrika?}

Mit diesem persönlichen Fazit schließt die Lehramtsstudentin Monika ihr Exkursionstagebuch ab. Die vergangenen zweieinhalb Wochen ist sie mit 15 Kommilitoninnen und Kommilitonen, zwei Dozierenden und einer begleitenden Forscherin durch den Südwesten Kenias gereist. Die Überblicksexkursion war auf die gegenstandsnahe Vermittlung von schulrelevanten geographischen Inhalten wie Wasserproblematik, Bildungssystem, Stadt-Land-Gegensätze, Segregation, Tourismus, Massai-Kultur, Vulkanismus und Vegetationszonen ausgerichtet. Gleichzeitig „prägt die Reise nach Afrika einen sehr, schenkt einem Erfahrungen, Erlebnisse \& neue Werte für das eigene Leben“, urteilt die Studentin Monika. Der Beitrag zeigt auf, inwiefern die Begegnung mit ,dem Fremden' im Kontext von Exkursionen dazu auffordert, eigene Selbst- und Welt-, d. h. ,Raumbilder' kritisch zu reflektieren, auf ihre Entstehung hin zu befragen und neu zu denken. Im gleichen Zuge fordert die Konfrontation mit den Welt- bzw. Raumbildern anderer dazu auf, deren Entstehung nachzuvollziehen und sie mit den eigenen in Bezug zu setzen. Einem humanistischen Begriff von Bildung folgend sind derartige Reflexionen von Selbst- und Weltverhältnissen ein Grundelement von Bildung an sich (vgl. Kokemohr 2007). Dass damit einhergehend die prinzipielle Konstruiertheit von Raumbildern zum Thema wird, zeigt, dass es sich um ein Kernanliegen geographischer Bildung handelt. In 


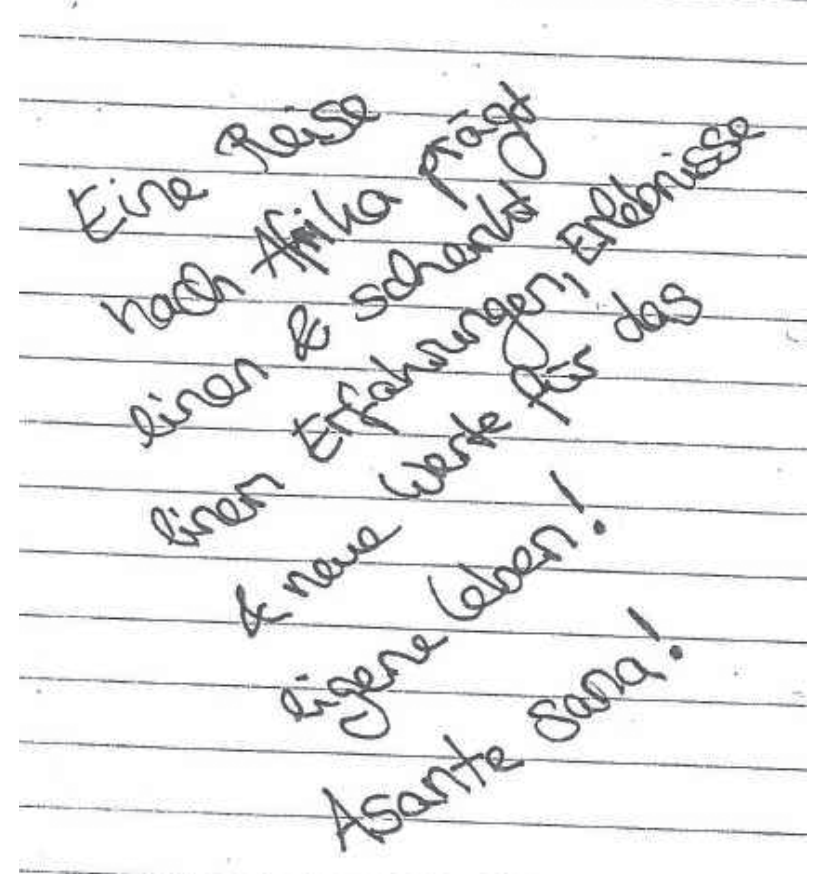

Abb. 1: Auszug aus Monikas Tagebuch (letzte Seite)'.

diesem Sinne gehen wir der Frage nach: Wie verdeutlichen Fremdheitserfahrungen die Konstruiertheit von Raumbildern? Dabei beziehen wir uns auf den Kontext der methodischen Großform Exkursion, die den Heimatraum verlässt und möchten empirisch fundiert die Relevanz von Exkursionen für die Professionalisierung angehender Lehrkräfte zum Thema machen.

\subsection{Zur Forschungsmethodik}

Im Sommersemester 2015 wurde die hier besprochene studentische Exkursion forschungsbasiert begleitet, um mittels ethnographischer Feldforschung der Frage nach Erfahrung und (Selbst-)bildungsprozessen auf Exkursionen nachzugehen. Die Datenerhebung bestand aus der Triangulation von teilnehmender Beobachtung, der Analyse privater Reisetagebücher und problemzentrierter Interviews. Die Datenquellen fokussieren damit unterschiedliche Umgangs- und Reflexionsformen mit dem Erlebten: Die teilnehmende Beobachtung zentriert die Prozesse von Praktiken und performativen Ausdruckqualitäten der Eindrücke (vgl. Gebauer \& Wulf 2003). Gerade die Forschung im Feld vermittelt das über das Faktische hinausgehende Wissen, denn erst durch leib-sinnliches (Mit-) Fühlen können die Forschenden an der Situation teilhaben, Unsichtbares und Unhörbares aufspüren und sich somit neue Verstehenshorizonte eröffnen

Zwei Hinweise: Die Namen wurden geändert; „Asantesana“ ist Kiswahili und heißt übersetzt „Herzlichen Dank“. (vgl. Abraham 2002, 186 ff.). Zudem finden verbale Reflexionen der informellen Gruppengespräche, wie auch der abendlichen durch die Leiter initiierten Gruppenreflexionen Eingang in die Forschung. Die Diskussionen zielten - ausgehend von subjektiv als auffällig, irritierend oder unklar Empfundenem - auf die intersubjektive und mehrperspektivische Analyse von vermeintlich ,Fremdem'. Die Untersuchung von zwei freiwillig bereitgestellten privaten Tagebüchern gibt hingegen Auskunft über Prozesse der zeitnahen und selbstbezüglichen Strukturierung von Gedanken, Problemen und Irritationen. Hier verorten wir mit Just (1996, 30) eine Kommunikationsstelle zwischen Innen- und Außenwelt: Das Selbst wird zum Betrachtungsgegenstand und damit das Tagebuch zum ,Spiegel der Seele und ,Spiegel der Welt'. Der Akt des adresssatenlosen Niederschreibens des subjektiv Empfundenen und Erlebten kann als Phase der intensiven Selbstreflexion, -aushandlung und -erkenntnis gedeutet werden. Fünf Wochen nach der Rückkehr wurden vier problemzentrierte Interviews geführt. Das Sampling folgte dem Prinzip der kontrastierenden Fälle. Indem in der Sozialforschung prinzipiell nie eine Gesamtheit untersucht wird, stellt sich in methodologischer Hinsicht die Frage, ob aus einer Auswahl auf generalisierbare Aussagen geschlossen werden kann. Eine Antwort lässt sich nur formulieren, wenn Klarheit darüber besteht, dass es letztendlich nicht um die Erhebung einer Absolutheit gehen soll, sondern die verschiedenen Ausprägungen des untersuchten sozialen Phänomens von Interesse sind. Der Akt des problemzentrierten, sprachlichen Vollzugs verkörpert eine Reflexion auf einer zeitlich distanzierten Ebene. Hier werden z. B. Irritationen, Bedeutungen oder Interpretationen dem/der Sprechenden erst bewusst und bedingen eine (erneute) Reflexion sowie neue Bedeutungen und Bezüge (vgl. Segbers \& Kanwischer 2015).

In diesem Forschungszusammenhang möchten wir Sie auf eine Reise nach Kenia mitnehmen und zwei exemplarische Situationen der Exkursion vorstellen.

\subsection{Situation 1: Der neue Wassertank der Ndauni Primary School}

Erst spät am Abend erreichen wir unseren nächsten Exkursionsstandort, eine ländliche Schule in der Nähe von Wamunyo im semi-ariden Hochland von Machakos. Es ist finster, als wir unsere Zelte aufbauen und bald darin verschwinden. Am nächsten Morgen werden wir überrascht davon, wo wir hier eigentlich sind. Wir kriechen aus unseren Zelten und werden dabei von hundert neugierigen Augenpaaren beobachtet. Sie schauen sich ganz genau an, was wir da treiben, wenn wir unsere Zähne putzen, die bunten Zelte abbauen, auf Klapphockern frühstücken. Sie 
wirken mächtig verwundert, über das, was da gerade auf ihrem Schulhof vor sich geht. Aber auch wir schauen nicht weniger verwundert $\mathrm{zu}$, was hier los ist: Die Kinder kommen näher und verstecken sich wieder, bringen Tische und Stühle heraus und bauen eine Art Aula auf dem staubigen Hof auf. Irgendwann werden wir tanzend abgeholt und finden uns plötzlich in einer Zeremonie wieder, in die das ganze Dorf involviert ist. Ein Verein aus Niedersachsen hat hier einen Wassertank gespendet (vgl. Stein 2013) und dies ist das Danksagungsfest. Singen, Tanzen und Vorstellungsrunden gehören zum fest etablierten Ritual. Jetzt auch für uns: Wir müssen mittanzen, ganz klar. Wir versuchen ihren schnellen Rhythmus zu halten, ihnen auch ein Lied zu präsentieren (auch wenn uns außer „Oh Tannenbaum“ wenig einfällt), jeder bekommt das Mikro in die Hand gedrückt und stellt sich vor. Eine absolut feierliche, bunte und herzliche Atmosphäre. Als dann die Kinder singen, Water is life ' und uns der simple Tank mit seiner enormen Wirkung erklärt wird, werden wir doch ganz schön stutzig. Hunderte von Tanzschritten, viele Dankesworte und drei Stunden später als geplant verabschieden wir uns und steigen wieder in unsere Jeeps.

Inwiefern verdeutlicht diese Situation die Konstruiertheit von Raumbildern?

,Übliche Afrikabilder ' konnten sich in dieser Situation bestätigen: Tanzende, singende, lebensfrohe Afrikaner/innen, bunt gekleidete Frauen, herzerweichend lachende Kinder passen genau in das verbreitete Afrika-Klischee. Und auch die Wassernot und der Mangel am Nötigsten sind leider in der Realität anzutreffen. Die Geschichte fügt sich fast schon zu perfekt in die vorgefertigten, von zu Hause über tausende Kilometer mitgenommenen Bilder. Auffällig ist auch geworden, wie die Einheimischen uns wohl sehen: Als die Reichen, aber Gnädigen aus einer anderen, heileren Welt in Europa. Interessant scheinen wir zu sein, man möchte unsere Haut berühren und mit uns auf Fotos abgelichtet werden. Irritiert sind wir zudem über die

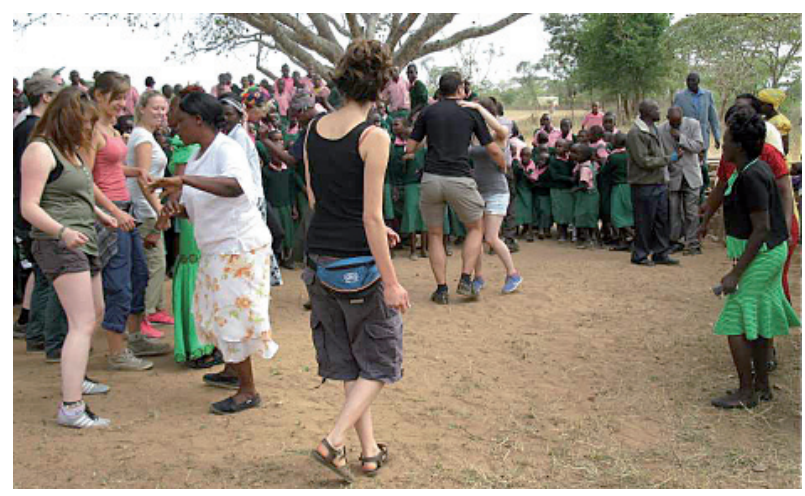

eigene Rolle. Vor einer Woche zu Hause noch ,arme Studierende', jetzt plötzlich, reiche Weiße', wenn auch in der Stellvertreterrolle für eine deutsche NGO als Spender eines Wassertanks, der die Lebensqualität dieser sympathischen Menschen erheblich verbessert. Die erlebte Diskrepanz zwischen deren Leben und dem eigenen, das ,beklemmende Gefühl' zusammen mit der ,ansteckenden Lebensfreude', die Monika selbst ,sehr glücklich fühlen lässt', sind wohl Aspekte, die die Situation in den Augen der Studentin zu einer ,prägenden Erfahrung' machen.

\subsection{Situation 2: Computer für die Grundschule in Ol Donyo Sabuk}

Wenige Tage später besuchen wir das Dorf Ol Donyo Sabuk, östlich von Thika. In Kleingruppen und gemeinsam mit einheimischen Jugendlichen besuchen wir verschiedene Schulen im Dorf. Zu dritt sind wir auf dem Weg in die Primary School. Dort zeigt uns der Schulleiter zunächst die Räumlichkeiten: Eine Schulküche, deren Kessel sie einer Spendenaktion aus Frankreich verdanken, einen Computerraum, in dem bislang lediglich ein paar Steckdosen vorhanden sind. Dann gehen wir in eine Schulklasse. Für die Kinder sind wir die absoluten Exoten. Sie wollen wieder unsere Haut anfassen, strömen zu Hauf auf uns zu. Die Nachbarklassen kleben an den Fenstern und Türen. Wir erzählen der Klasse etwas von unserer Heimat, über die Jahreszeiten, das Klima, den Fußball und beantworten Fragen der Kinder. Dann mischt sich der Schulleiter ein und sagt, dass das Land, wo wir herkommen, sehr, sehr reich sei und dass wir drei, die hier stehen, wiederkommen und die Computer für die Schule mitbringen werden. Die Kinder fragen weiter nach unseren Eltern und den deutschen Fußballstars. Im Lehrerzimmer fordert uns der Schulleiter erneut auf, wiederzukommen und die Computer mitzubringen. Ein langes Gespräch beginnt: Wir erzählen ihm, dass wir selbst Computer haben, aber nicht einen ganzen Schulsatz kaufen und ohne Weiteres noch mal vorbeikommen könnten. Dass wir für die Reise hierher selbst lange sparen mussten und dass es auch im ,reichen' Deutschland viele Menschen gibt, die Verlierer/innen im ,Gewinnerland' sind und um ihr Sattwerden bangen müssen. Und wir erzählen

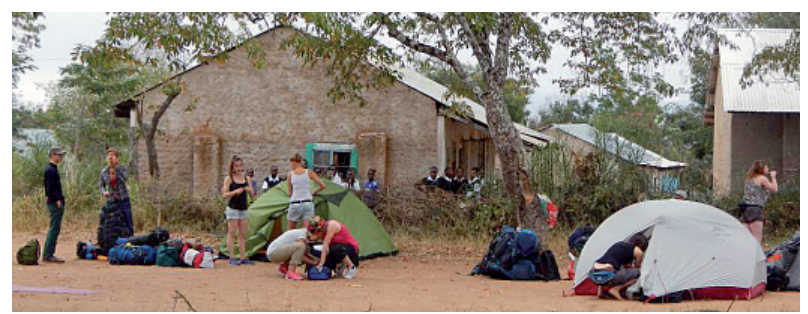

Abb. 2 und 3: Zu Gast an der Ndauni Primary School (eigene Aufnahmen). 


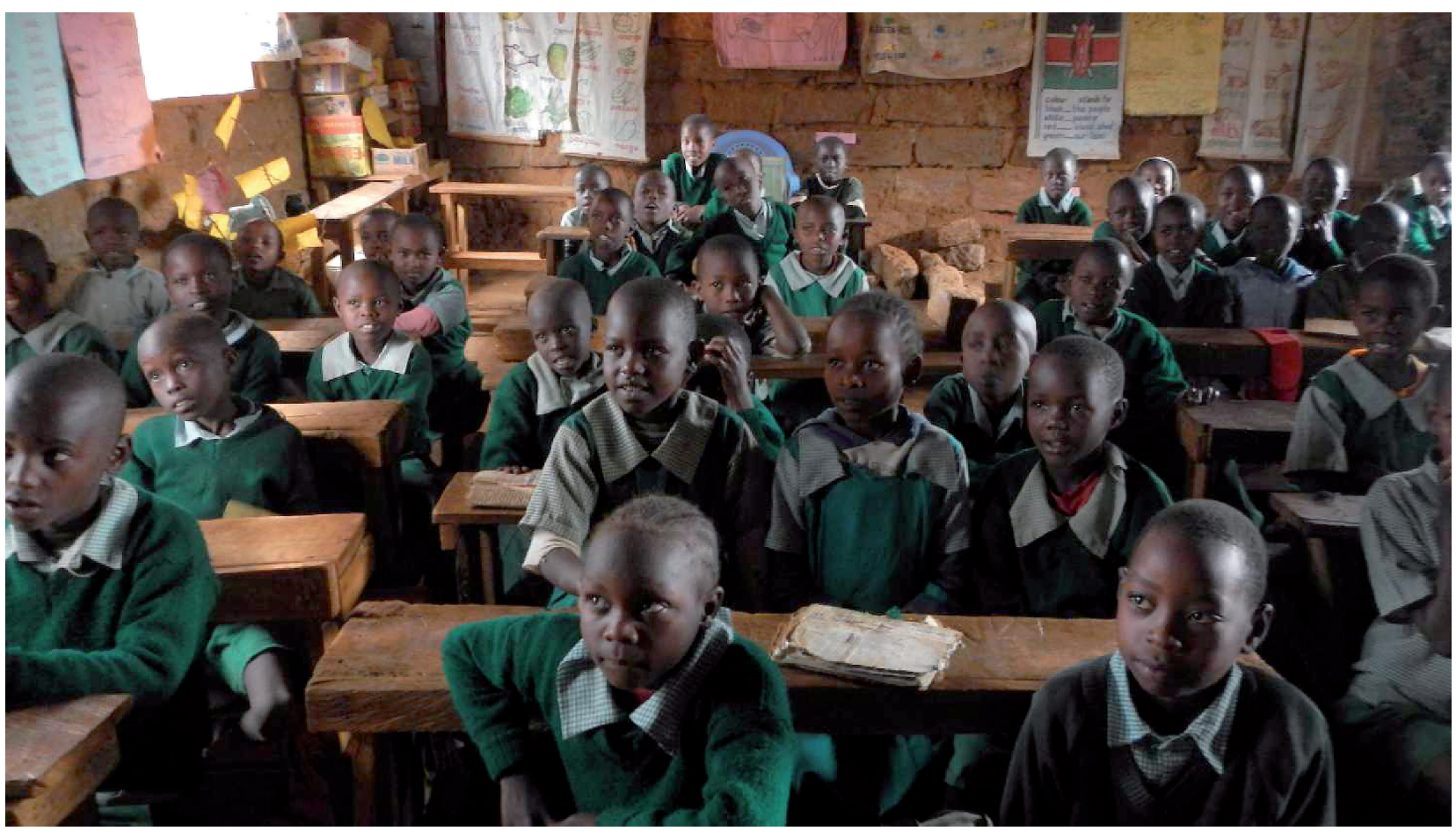

Abb. 4: Ein Besuch an der Primary School Ol Donyo Sabuk (eigene Aufnahme).

ihm von der Situation in der EU. Deutschland ist Gewinner, Griechenland Verlierer. Selbst in Europa gibt es Armut. Und Afrika? Europa, der Gewinner, wohingegen Afrika verloren hat? Der Schulleiter ist höchst irritiert. Er sagt, das habe er alles nicht gewusst und nie gedacht. Bei der herzlichen Verabschiedung schauen sich nun ziemlich verdutzte Augenpaare an. Mit einem sehr verstörten und beunruhigten Gefühl verlassen wir - umringt von zig Kindern - den Schulhof. Als wir die anderen aus unserer Exkursionsgruppe wiedertreffen, ist der Redebedarf riesig.

Inwiefern verdeutlicht diese Situation die Konstruiertheit von Raumbildern?

Wahrscheinlich sind wir in dieser Situation nicht weniger als der Schulleiter vor den Kopf gestoßen. Wo ist all diese Herzlichkeit, Lebensfreude und der feierliche Empfang, den wir so sehr zu unserer Begeisterung an der letzten Schule erlebten? Mit dem erst kürzlich gewonnenen Bild von der kenianischen Schule muss zwangsläufig binnen Minuten gebrochen werden. Wie reagieren wir am besten auf die Not der Menschen hier, wie auf die Forderung nach den Computern? Der Mangel der Menschen hier, Missverstehen und Fragezeichen rücken in den Vordergrund. Den Kindern wird ihr Bild von den hellhäutigen Reichen wohl bestätigt bleiben, doch nicht als gnädige Spender, sondern wohl eher als Voyeure und egoistische Touristen. Die hautnahe Konfrontation mit der Situation der Besuchten geht uns bis unter die Haut. Mit unserer eigenen Situation werden wir dabei nicht weniger konfrontiert: Schließlich haben wir viel mehr als die Menschen hier. Sind wir nun ,Reiche? Wir würden gerne helfen, könnten doch auch mehr tun, als den Satz Atlanten zu schenken, den wir dabei hatten, verfluchen diese von Disparitäten geprägte Welt. Wieso haben wir keinen Satz Computer? Und wie ist das eigentlich mit den Leuten, die vor meinem Stammbäcker auf der Straße sitzen und betteln müssen? Was bedeuten die Meldungen, die uns aus Griechenland und von den europäischen Außengrenzen erreichen? Wie lassen sich die erlebten unterschiedlichen Wahrnehmungen von sich selbst und den anderen erklären und deuten? Der Schulleiter wird durch unser Gespräch damit konfrontiert, dass sein Bild von Europa nicht ganz stimmig ist. Gleichzeitig ist die Not hier keineswegs zu leugnen. Die Studierenden werden damit konfrontiert, dass sie selbst Computer usw. haben und sie irgendwie auch etwas spenden könnten. Die Studierenden erkennen ihre ,eigene Verwobenheit in Machtverhältnisse" (Schröder 2016, 23) und beginnen, „Formen nicht bewusst-intentionaler Machtausübung" (ebd.) zu hinterfragen. Der Fokus wird also erweitert, „indem nicht länger nur die Anderen zum Thema werden, sondern auch die Kategorie des ,Eigenen' in ihren relationalen Bezügen in den Blick rückt. Es geht dann auch darum, die privilegierte Situiertheit derjenigen zu thematisieren, die in Deutschland als legitim zugehörig gelten" (ebd., 24). In der Reflexion wird deutlich, dass es im Sinne interkulturellen Lernens der „Dekonstruktion kulturalisierender 
Deutungs- und Legitimationsmuster von sozialer und politischer Ungleichheit“ (Mönter 2013, 95) bedarf. Erstens: So, wie unsere Weltbilder nicht der Realitäten vor Ort entsprechen - trotz der vielen Informationen die wir Büchern, TV und Internet entnehmen können - sind auch die Weltbilder anderer verzerrt. Zweitens: Eine Diskussion kapitalistischer Machtstrukturen ist unabdingbar, „denn genau dort, wo die expandierende Herrschaft des kapitalistischen Imperiums auf andere ,Rassen' getroffen ist, hat sich der Rassismus als eine Form der Ausschließungspraxis entwickelt" (Hall 2000,10). In dieser Erkenntnis liegt auch eine Antwort auf die Armut vor Ort. Das Bewusstsein über das eigene Eingebundensein in diese Strukturen gilt es zu erzeugen, verantworten und auszuhalten.

\section{2 ,Afrika'-Bilder und Raumkonstruktionen im Diskurs}

„Die einseitige Konzentration auf strukturelle Aspekte von begrenzten Raumausschnitten vernachlässigt das vielfältige und komplexe Zusammenspiel von nicht quantitativ messbaren Faktoren sozialräumlicher Prozesse, insbesondere, wenn Räume an sich und nicht die bedeutungsvolle Aneignung von Raum im Vordergrund stehen - anders gesagt, wenn also beim Raum und nicht beim Sozialen angesetzt wird. So ist die Art und Weise, wie Menschen räumliche Gegebenheiten nutzen, zentral für das Verständnis sozialer Räume“ (Schlottmann et al. 2014, 100). Die o. g. Beispiele von Erlebnissen im Rahmen einer geographischen Exkursion machen dies deutlich: Auch wenn die Schwerpunkte des Exkursionsprogramms zunächst ein wenig an das klassische Betrachten im Sinne von quantitativ zählbaren ,Raumausstattungsmerkmalen' erinnern mögen, zeigen die Beispiele in Kapitel 1, dass Räume „als Ausdruck und Konsequenz gesellschaftlicher Praktiken und Strukturen gedacht - als sozial konstruiert" (Glasze \& Mattissek 2009, 40) verstanden werden müssen. Um - bezogen auf dieses Verständnis - soziale Räume verstehen zu können, bedarf es daher realer Begegnungen vor Ort. Durch Begegnungen im Realraum kann unmittelbarer erfahren werden, „wie Raum in kulturellen Praktiken über Handlung erst erzeugt wird“ (Dickel 2006, 10). In Bezug auf Repräsentationen von ,Afrika'-Bildern wird so ein Bewusstsein angebahnt, das zeigt, „spaces and places are always and already works-in-progress" (Horton \& Kraftl 2014, 275). Es gibt also nicht dieses oder jene Bild von ,Afrika', sondern die Repräsentationen sind mannigfaltig. Dies gilt auch für Räume in kleinerem Maßstab. Der Raum als place ist also immer ein Produkt handelnder Akteure: „All sorts of people, things and ideas from all sorts of places make them (local places) what they are" (ebd., 279). Vor diesem Hintergrund erscheint es als Herausforderung, ,Afrika', bzw. in kleinerem Maßstab etwa das Alltagsleben in einem Dorf im ländlichen Raum Kenias, im Schulunterricht angemessen zu thematisieren. Eine räumlichthematische Auswahl ist reflektiert zu treffen, da stets die Gefahr einseitiger Vereinfachung bzw. einer unzureichenden Repräsentation der Diversität räumlicher und kultureller Aspekte besteht, denn „Afrika' kann es nur in einem doppelten Plural geben. Einerseits im Plural der multiplen Realitäten in Afrika und andererseits im Plural der multiplen Perspektiven auf Afrika“ (Kersting 2011, 3).

Ferner wird gerade im Zusammenhang mit dem Raumbeispiel ,Afrika' deutlich, dass Vorurteile und Stereotype, aber auch bisweilen rassistische Äußerungen über geographische Räume und ihre Menschen allgegenwärtig sind (vgl. Reinfried 2015a, 74). Für den Schulunterricht ist diese Ausgangssituation im Kontext der didaktischen Rekonstruktion bedeutsam: Ein nach diesem Modell konstruierter Unterricht verfolgt das Ziel, „die bestehenden Vorstellungen in neue Vorstellungen zu verwandeln, sie im Neuen aufzuheben. Didaktische Rekonstruktion heißt, Schülervorstellungen und fachlich geklärte Vorstellungen systematisch so aufeinander zu beziehen, dass es den Lernenden möglich wird, Hindernisse des fachlichen Lernens zu überwinden, ihr Vorwissen anzureichern und umzustrukturieren und einen Perspektivwechsel vorzunehmen" (Reinfried 2015b, 80). Die Afrikabilder der Lernenden betreffend bedeutet dies, dass es der Erkenntnis des Konstruktionscharakters dieser Bilder und Vorstellungen mit einer Dekonstruktion selbiger in der Folge bedarf. Damit dies wirksam gelingt, ist neben einem klaren Perspektivwechsel auch eine Enthierarchisierung (im Hinblick auf zugrundeliegende Interessen und Machtverhältnisse) notwendig, denn "it is important to bear in mind that it is not enough to deconstruct, one must decolonize at the same time. Deconstruction without decolonization within the context of critical anti-colonial work has only limited potential for transformation" (Sefa Dei 2012, 61). Es bedarf also des Aufgreifens postkolonialer Ansätze, um bestehende Denk- und Handlungsmuster zu reflektieren und aufzubrechen (vgl. Lossau 2012). Um Lehramtsstudierende mit dieser Herausforderung vertraut zu machen, bedarf es also einer kultursensiblen Ausbildung, die im Sinne einer Metareflexion hinterfragt, inwiefern „Kultur - auch die eigene - das Denken und Handeln beeinflusst" (Reinfried 2015a, 76). Durch derartiges Reflektieren soll hinterfragt werden, $\mathrm{ob}$ „neue Informationen entsprechend der bereits im Gedächtnis vorgenommenen Einteilungen geordnet werden" (ebd.). Es gilt danach zu fragen, ob ,Fremdheit' als etwas Unbekanntes, aber dadurch irgendwie 


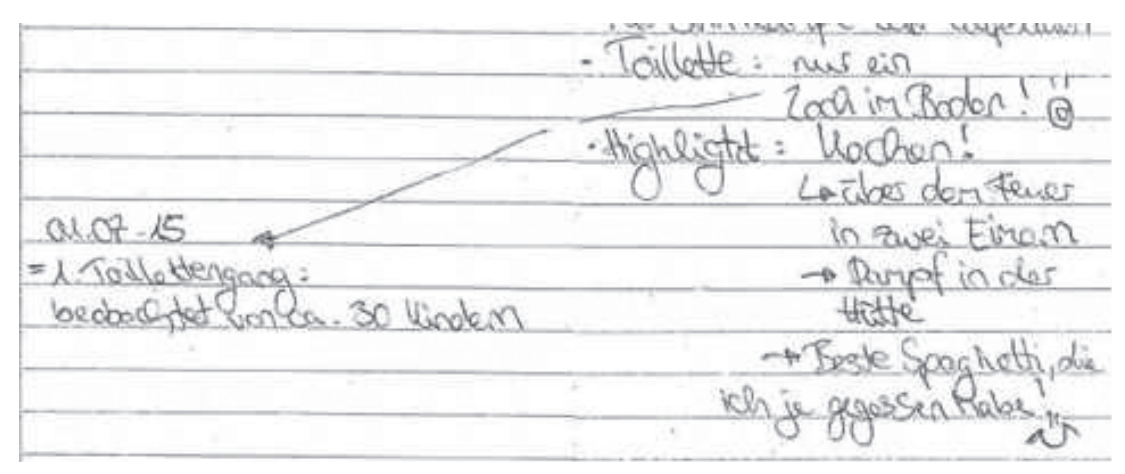
Abb. 5: Auszug aus Monikas Tagebuch.

subjektiv Berührendes erlebt wurde und als durch gesellschaftliche Diskurse geschaffenes erkannt wird (vgl. Hall 2000). Gerade die bestehenden Einteilungen können so aufgebrochen und eine neue Stufe interkulturellen Bewusstseins erreicht werden. Konkret wird dabei von einem geographischen Kulturverständnis ausgegangen, welches Kultur definiert als ,a particular way of life, such as a set of skilled activities, values, and meanings surrounding a particular type of practice" (Knox \& Marston 2016, 180). Um eine bewusste Wahrnehmung der Darstellung Afrikas zu erreichen, muss also zunächst bei der eigenen (Raum-)Wahrnehmung angesetzt werden (vgl. Kersting 2011,3). Die methodische Großform der Exkursion ist prädestiniert dafür, denn die Erfahrung vor Ort ist unmittelbar körperlich und emotional grundiert. Es gibt kein Entrinnen. „Im Gelände schlägt das Herz der Geographie“ (Falk 2015, 150) - in der unmittelbaren Begegnung mit dem Gegenstand des Fachs liegt das körperliche und emotionale Zentrum. So wird erfahrbar, „dass Menschen aufgrund ihrer eingeschränkten Wahrnehmung und Bewertung keine unmittelbare und ,objektive' Information über die sie umgebende Welt haben, sondern dass ihre Alltagswahrnehmung durch kollektive und subjektive Vorstellungsbilder angeleitet wird“ (Reuber 2016, 111).

\section{Zu Exkursionen und ihrer Wirkung}

„Wenn sich eine fremde Logik festsetzt, wenn einem die gewohnheitsmäßigen Überzeugungen und Werte unversehens zu Kleinholz geschlagen werden, dann nennt man die Erregung, die einen dabei überkommt, Kulturschock" (Bohannan \& van der Elst 2003, 90).

Gerade Exkursionen bieten mehr als jede andere Form von Lehrveranstaltung das Potenzial, einen eben solchen ,Kulturschock' zu provozieren. Dieser Form des Schocks kommen dabei allerdings keine rein negativen Eigenschaften oder Auswirkungen zu. Vielmehr birgt eine Irritation, die zum Anlass der Reflexion wird, große Bildungspotenziale. Dies sei im Folgenden anhand einiger exemplarischer Beispiele der Exkursion nach Kenia vertiefend aufgezeigt.

\subsection{Reisende Irritation - mitreißende Irritation}

Stets reisen wir mit vorgefassten Erwartungen durch die Welt, ob im heimischen Umland oder im fernen Afrika. $\mathrm{Da}$, wo wir unsere vertraute Welt ein Stück weit verlassen - so wie auf einer Exkursion nach Kenia - werden die als ,Afrika'-Bilder im Kopf konservierten Erwartungen mit der Wahrnehmung des konkreten Raumausschnitts konfrontiert. Es ist kaum abzuwenden, dass Unvorhergesehenes und Überraschendes mit den vorgefassten, erwarteten Mustern, Erwartungen und Bildern bricht und zwar immer abhängig von jedem einzelnen subjektiven Muster, Erwartungshorizont, Bild.

Das Datenmaterial zeigt, wo sich für die einzelnen Teilnehmenden plötzlich ein gewisser Bruch auftut: Das können im Alltag ganz ,kleine' und selbstverständliche Dinge sein, wie unsere Hygiene- und Kochgewohnheiten, die auf einmal keine Allgemeingültigkeit mehr haben, sondern zu einer höchst ,besonderen' Situation werden (siehe Abb. 5):

Entgegen der Darstellungen ihrer Kommilitoninnen und Kommilitonen (siehe Kap. 1) ist die gleiche Begegnung an der Schule für Jana ausdrücklich negativ behaftet, wie sie es im Interview formuliert:

"WAS ich oder was ich SCHWER fand wirklich
oder für mich persönlich KEIN schönes Erlebnis,
sage ich mal, war wo wir den ersten Tag in der Schu-
le waren und äh alle da mit dem Wassertank ähm
(?), halt dieses Water is Life. Und dann gehen wir
den nächsten Tag zu diesen Thomson-Falls, oder wie
die heißen, und äh, dort ist dann die Müllkippe di-
rekt im Wasser. Das waren so zwei Sachen, die nicht
zusammengepasst haben"(Jana)

Jana reibt sich an Widersprüchen auf, dass wir eine Nacht später in einem schicken Hotel mit reichlich Wasser nächtigten und dass die hier so kostbar erlebte Ressource Wasser anderswo ganzanders gehandeltwird.

Die Texte der Studierenden zeigen ausdrücklich, dass die Situation am Wassertank eine Konfrontation mit der eigenen Rolle bewirkte. Jana beschreibt ihr Verhältnis dazu, dass sie eigentlich nur eine andere Deutsche, nicht aber die tatsächliche Spenderin des Wassertanks ist: 
„Was auch/so auch erstmal so ein Moment Mal. Wieso sitze ich jetzt hier? Bis hin zu ok, das wird schon in Ordnung sein, dass man das jetzt stellvertretend annimmt" (Jana)

Was sich in der Situation ereignet, geht über rein rationales Erfassen hinaus, sondern geschieht auf beiden Seiten, auf Seiten der Besuchten und der Besuchenden über sinnliches Fühlen und leibliches Spüren:

\begin{abstract}
"Gerade die Kinder in der Schule fanden das ja auch ungeheuer spannend dann/wollten mal anfassen/äh/Ich selbst habe dann natürlich auch mal angefasst, so über die Haare gestreichelt oder über die Hand so und die Haut war auch (.) ANDERS WEICH, aber auch total weich. Und die schönen krausen Haare und für die war es natürlich ganz faszinierend" (Jana)
\end{abstract}

Die Erfahrungen, die so gemacht werden (können) sind in diesem Sinne eine ganz besondere menschliche Möglichkeit des Erkennens, die nicht dasselbe wie Denken oder Erleben ist. Mit Meyer-Drawe (2008) und Tengelyi (2002) gehen wir davon aus, dass erst da, wo etwas Neues, Unvorhergesehenes, ja Überraschendes geschieht, von ,Erfahrung' gesprochen werden kann. Daher kann die Erfahrung nicht einzig auf das Bewusstsein zurückgeführt werden, sondern deutet „auf eine Bruch- oder Rissstelle im Bewusstsein hin“ (ebd.): In der Erfahrung zerspringt die Intention des Bewusstseins, „indem sie von der Welt überrascht und beschlagnahmt wird“ (Meyer-Drawe 2008, 188f). Diese Überraschungen der Welt sind es, die als ein fremder Anspruch auftreten, durch den das Bewusstsein „wie durch eine Ohrfeige getroffen werden kann“ (ebd., 189). Es ist also nicht die Sache an sich - die Infrastruktur/sanitären Anlagen, das kenianische Kind, der Wassertank - sondern das Fremde daran „sucht uns heim, und versetzt uns in Unruhe, noch bevor wir es einlassen oder uns seiner zu erwehren trachten" (Waldenfels 1997, 42). Dem Fremden kommt dabei nicht der Status des Passiven zu, uns entzieht sich hier etwas. Etwas Unbestimmtes, das noch nicht als etwas gedeutet werden kann. Dieses Sich-Entziehen des Fremden ist das Aktive, das vom Fremden selbst ausgeht. Es ist sein Anspruch, „als etwas, das an uns gerichtet ist" (vgl. Koller 2012, 162). Das Fremde fordert in dieser phänomenologischen Perspektive dazu heraus, in eine Beziehung mit ihm zu treten und beides - Eigenes und Fremdes - zum Gegenstand der Betrachtung zu machen: Es weckt die Aufmerksamkeit und die Bereitschaft zu bewusster Sinnestätigkeit, denn vorhandene Sinnbezüge sind gestört und Regelsysteme gesprengt (vgl. Waldenfels 1997, 52). Man macht Erfahrungen mit der Welt, denn das Neue solcher Weltbegegnun- gen kann zunächst „nicht in die Grundfiguren jener lebensgeschichtlich aufgebauten Ordnung integriert werden (?), die meine alltägliche Interpretation leiten“" (Kokemohr 2007, 14)., Welt', also das, was der besuchte Raumausschnitt zeigt und an Überraschungen und Beschlagnahmungen öffnet, kann als Metapher für das Fremde, das sich der Aneignung entzieht, ausgelegt werden (vgl. Dörpinghaus 2009, 39).

Wo vorher fraglose Selbstverständlichkeit von Ausschnitten der Welt war, bringt die über alle Sinne und über das gesamte leibliche Sensorium wahrgenommene Irritation so einen Unterschied ins Spiel: Als Exkursionsteilnehmende/r war man doch gewohnt, sich zurücklehnen zu können - mitschreiben ja, aber tanzen? Was ist hier los? Was macht mein Gefühl von kitzelnder Spannung einerseits und bedrückender Beklemmung andererseits aus? Welche Rolle habe ich hier plötzlich - die Kinder wollen mich anfassen, ich bin Exot/in, besonders, mittendrin in ,Afrika', letzte Woche war ich noch, arme/r Student/in', nun ,gnädige/r Weiße/r' oder, geizige/r Weiße/r'? Welche Vorstellungen des Anderen stecken dahinter? Womöglich war auch der Schulleiter der zweiten Schule getroffen von unseren erschrockenen Gesichtern und von unseren Erzählungen.

Was passiert also in den in Kapitel 1 beschriebenen Situationen mit den mitgebrachten Selbst- und Raumbildern? Das Fremde in den Konfrontationen mit einer ungeahnten eigenen Rolle und einer individuell auf unterschiedliche Weise überraschenden Situation verwandelt das zuvor gegebene subjektive Bild von Afrika wie durch eine, Ohrfeige' in ein Problem. Noch offensiver formuliert: Die Person wird zu einer Umstrukturierung ihres Vorwissens genötigt, statt in ihren alten Gewohnheiten bekräftigt (vgl. Fromme 2001, $421 \mathrm{ff}$.). Was merkwürdig klingen mag, folgt jedoch einem pädagogischen Konzept, das davon ausgeht, dass es ohne die Triebfeder der Irritation überhaupt keine ,echten Erkenntnisse', sondern nur „gedächtnismäßige Einprägung überlieferter Sinngehalte" gibt (Copei 1969, 60; 102 zit. in Fromme 2001, 422). Rhode-Jüchtern \& Schneider $(2012,148)$ betonen in diesem Sinne, dass ein Problem ohne Verunsicherung als solches überhaupt nicht wahrzunehmen, geschweige denn, zu bearbeiten sei. Es wird davon ausgegangen, dass das Verblüffen am Phänomen (bestenfalls) in eine staunende Fragehaltung mündet, die auf Bestimmung desselben zielt. Dickel \& Schneider (2013) sprechen an dieser Stelle von, echten' Fragen, die mit den Schemata bestehender Erfahrungen bzw. Vorstellungen im Kopf nicht beantwortet werden können und Selbst- und Weltbild daraufhin geändert werden müssten. Unverhoffte Begegnungen können so die Öffnung zu einer Welt provozieren, die Antizipationen, Erwartungen, ,Afrika'-Bilder zeigt, die 
zuvor unbemerkt in Geltung waren. Darin steckt, dass jede Erfahrung auf sich selbst zurückgeworfen wird (vgl. Meyer-Drawe 2008, 189). Erst aber diese Rückwendung der Erfahrung auf sich selbst trägt die eigentlich belehrende Kraft der Erfahrung in sich (vgl. Buck 1989, 3). Die neuen Sichtweisen, die aufzutauchen vermögen, müssen nicht bereits ,fertig' sein. Vielmehr muss der zunächst probeweise verfolgte Weg noch geprüft, hinterfragt, reflektiert werden, um das Erkannte genauer zu fassen.

\subsection{Von der Reflexion der Irritationen zur Reflexivität}

Die irritierenden Konfrontationen mit Fremden bzw. Fremdem, wie sie sich in den hier dargestellten Situationen vollzogen haben, können als typische Aufforderungen gelten, Erwartungen des jeweiligen Bewusstseins, zu Bruch zu bringen. Allerdings ist die Art und Weise des Umgangs mit dem Fremden entscheidend dafür, ob die Konfrontation ,angemessen, produktiv oder reich ist und den Namen von Bildung zu Recht trägt. Das entscheidet sich erst in der Geschichte seiner Fortsetzungen" (Kokemohr 2007, 26). Waldenfels (1997, 37 ff.) zeigt drei Möglichkeiten auf, mit dem Fremden als Stifter von Unruhe umzugehen: Die erste ist ein xenophobes Ausweichen, d. h. Fremdheit wird mit Feindlichkeit gleichgesetzt. Die Eigenheit des Eigenen wird nicht einer Befragung unterzogen, in dem Sinne, dass ,ich mir und wir uns als Selbst gegenübertreten und uns das Eigene als Eigenes begegnet" (ebd., 46). Stattdessen wird das Eigene zur Instanz der Deutung und der Entscheidung über Freund/in oder Feind/in und der Auseinandersetzung mit ihm ausgewichen. Hätten wir den Schulleiter der zweiten Schule für überheblich in seiner Forderung nach 26 Computern gehalten und als völlig vermessen abgetan, so hätten wir auf diese Weise mit der Konfrontation mit einer fremden Vorstellung geantwortet.

Die zweite Form ist eine Bändigung der Fremdheit. Der Umgang mit dem Fremden ist so, dass „Fremdheit [...] mit einem Makel behaftet [scheint], den es zu tilgen gilt" (ebd., 49, Änderung d.A.). Es handelt

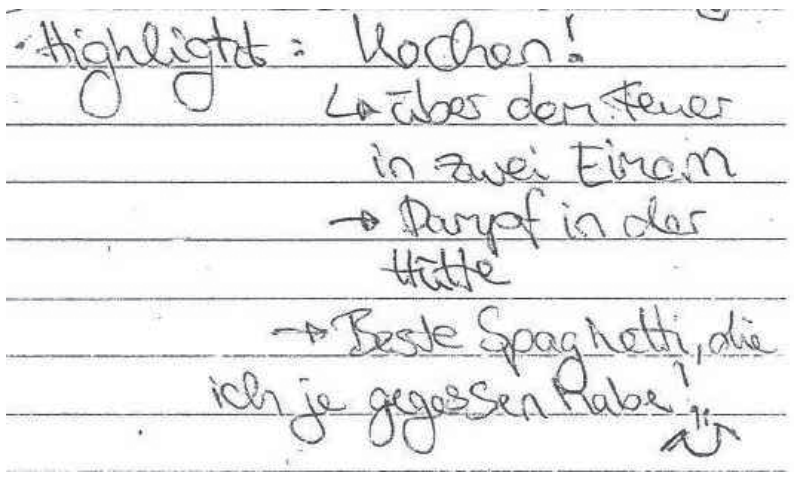

Abb. 6: Auszug aus Monikas Tagebuch. sich um den Versuch, Fremdes in die eigene Ordnung $\mathrm{zu}$ integrieren, wobei eigene Vorstellungen, Bilder, Ideale, Werte als fraglose Normen gelten. Im Repertoire der Erinnerungen und bekannten Inhalte wird nach Erklärungen gesucht, bis Differenzen verblassen, bis das Fremde seiner Fremdheit beraubt ist. Einer Irritation der bestehenden Ordnungsfiguration wird sich verwehrt (ebd., $60 \mathrm{ff}$.). Resultat sind Zentrierungen wie Ethno-, Ego- oder Eurozentrismus. Hätten wir geurteilt, der Schulleiter sei eben ein typischer, ,armer' und ,bettelnder Afrikaner' und uns damit die Begegnung erklärt, so wären wir auf diese Weise mit der Situation umgegangen.

Hätte Monika das Erlebnis des „exotischen Kochens" lediglich als ein spannendes Reiseerlebnis verbucht, dass man den Daheimgebliebenen erzählen und seine Erlebnisse der ganz besonderen Art demonstrieren kann, so hätte sie auf diese Weise auf den Anspruch des Fremden geantwortet. Allerdings zeigt u.a. die folgende Interviewsequenz, dass Monika ihre und die kenianischen Alltagsbedingungen der Reflexion unterzogen hat:

"Ich war alleine zu Hause und ich habe echt versucht, alles Mögliche aufzuessen oder noch mitzunehmen in den Urlaub und dann meine Schwester, ja das hast Du doch früher nicht gemacht. Ich so, naja, aber wenn man das jetzt mal GESEHEN hat wovon andere so leben und was wir dann dagegen für einen Luxus in Deutschland haben" (Monika)

Sie hat das Bild von Lebensfreude der Kenianer und alte Normen des Alltagshandelns der Revision unterzogen und geändert. ${ }^{2}$

Damit kommen wir zur dritten Art des Antwortens auf den Anspruch des Fremden. Diese ist eine Änderung der eigenen Einstellung. Dies erfordert, nicht selbst den das Fremde bestimmenden Gültigkeitsanspruch zu erheben, sondern als Subjekt eine nachrangige Position einzunehmen. Vorgelagert ist der Anspruch, der vom Fremden ausgeht, dem nicht ausgewichen werden kann. Eigenes und Fremdes werden zueinander in Beziehung gesetzt. Damit öffnet sich die Sphäre des ,Zwischens'. Neuartige Gedanken, die auf diese Weise entstehen, sind weder Geschöpfe des Subjekts noch des Anderen. Sie entstehen zwischen beidem (vgl. Waldenfels 1990, 50 ff.). In solchen produktiven bzw. kreativen Antworten wird Neues auf synergetische Weise hervorgebracht.

Folgende Interviewsequenz zeigt ein solches, Neues schaffendes Antworten von Peter im Kontext der Situation am Wassertank:

2 Wie lange dies anhält, darüber können wir auf dieser Studie basierend keine Aussagen treffen. 
"Also man war ja noch gar nicht richtig angekommen, man weiß das alles noch gar nicht richtig einzuschätzen. Und gekippt hat das dann in dem Moment, als wir gemerkt haben, dass wir da gerade dieses DING einweihen. Am Anfang wussten wir das nicht so richtig einzuschätzen, hätte ja sein können, dass jeder Weiße so begrüßt wird in jedem Dorf oder so das war uns ja auch nicht klar" (Peter)

Peter beschreibt aus seiner Perspektive einen Anspruch des Fremden, der sich an ihn stellt: Er kennt die Gepflogenheiten in der besuchten Kultur nicht und kann das, was er erlebt, nicht hinsichtlich der zugrundeliegenden Bedeutungen einordnen, lediglich vage Vermutungen anstellen. Dass er von, wir" spricht zeigt, dass es seine Einschätzung auf die der anderen überträgt. Damit ist eine Trennung in die Gruppe der einen (Deutschen) und anderen (Kenianer) vorgenommen.

„Aber dass das so eine Ausnahmesituation darstellte, das hab ich erst etwas verspätet begriffen und $A B$ DEM Moment da habe ich mich auch mal kurzl kurz schlecht gefühlt"(Peter)

Hier legt Peter offen, dass im Moment des Auffällig-Werdens etwas mit seiner Wahrnehmung nicht stimmt und sich ein Riss in seinem Bewusstsein vollzieht. Hier zerspringt seine Möglichkeit der Einordnung des konkreten Erlebens unter bereits bestehende Vorstellungen - das Bewusstsein wird „wie durch eine Ohrfeige getroffen" (Meyer-Drawe 2008, 189). Ein Ausweichen, nicht zu reagieren, ist nicht möglich. Das affektiv-emotionale Spüren gerät stattdessen in den Vordergrund. Die Sphäre des ,Zwischen' ist geöffnet. Mit Rückgriff auf das Reflexionsgespräch an diesem Abend zeigt Peter, dass er Eigenes und Fremdes zueinander in Beziehung gesetzt hat: Eigene Vorstellungen von den Gepflogenheiten in Kenia, eigenes Empfinden von zunächst Freude und ,Spaß` und dann sich „schlecht gefühlt" zu haben einerseits und der Konfrontation mit fremden Vorstellungen, Wünschen, Bedeutungen, Hoffnungen und Praktiken andererseits.

„Da haben wir ja in der Reflexion drüber gesprochen, welche Gefüble überwiegen: Entweder ich hab damit ja eigentlich gar nichts zu tun und ein schlechtes Gewissen. Oder aber: Die freuen sich einfach, dass überhaupt mal jemand da ist und man sich mal bedanken kann und sie 'nen Ansprechpartner haben. Und dann nehmen wir diese Rolle auch sehr gerne ein auch wenn wir wissen, dass wir damit nicht so viel zu tun haben. Wenn wir das einzuordnen wissen, ist da ja legitim, denke ich"(Peter)
Peter hat seine eigene Rolle, Empfindungen und Vorstellungen mit dem, was ihm als fremd entgegentrat, neu ausgehandelt. Zwischen beidem hat er eine neue Perspektive auf sich und die anderen gewonnen. Er hat sich in die Gastgebenden hineingespürt, die Trennung zwischen , die ${ }^{6}$ und ,wir ${ }^{`}$ ein Stück weit aufgehoben. Damit hat er transkulturelle Sensibilität gezeigt oder vielmehr entwickelt. Es ist also „die Weise unseres Nachdenkens, die darüber entscheidet, wie sich uns eine Sache darstellt" (Dickel 2016, 48).

Neue Grundfiguren subjektiver Selbst- und Weltverhältnisse und neue Bilder von bestimmten Raumausschnitten entstehen erst in solchen kreativen Antworten. So auch die Einschätzung von Koller (2012) und Kokemohr (2007). Wenn das Fremde und die durch selbiges ausgelösten Irritationen didaktisches Mittel sein soll, um neue Zwischenräume zu eröffnen, die Bilder von Räumen transformieren, muss auch das Geworden-Sein von alten und neuen, von eigenen Perspektiven und Perspektiven anderer verstehbar gemacht werden. Es braucht Reflexion davon, ob uns manche(s) bereits als, Fremde(s) 'vermittelt wird, mit einem mitunter rassistischen Kern. Und es braucht die Reflexion davon, was wir denn da eigentlich tun, wenn Vertrautes fraglich wird.

Die Außeralltäglichkeit der Lehr-Lernsituation Exkursion macht Konfrontationen mit dem Fremden wahrscheinlich. Beunruhigungen bestehender Ordnungen, wie in den geschilderten Situationen, geht bereits die Aufforderung der reflexiven Verarbeitung voraus. Reflexionen finden aufgrund der Beunruhigungen im informellen Rahmen statt, wie beim Tagebuchschreiben.

„An dem Abend hab ich SO viel Tagebuch geschrieben. Das war/das war (..) schön. Da hab ich ganz viel für mich selbst auch noch mal reflektiert. Ich brauch dann ab und zu auch mal Momente der Ruhe, wo ich dann auch mal für mich bin, um/um Dinge auch wirklich einschätzen zu können"(Peter)

Reflexionen innerhalb der Gruppe finden auch gerade dann statt, wenn kein offizielles Programm geplant ist, wie beim Kochen, am Lagerfeuer, während des Weiterreisens, wie die Daten an vielen Stellen zeigen. So z. B. Boris:

"Es ja wirklich meistens so ne, wenn man irgendetwas erlebt hat und dann danach wieder im Jeep und so hat man dann/ja erstmal darüber geredet und so. Und ich fand das hat auch aufjeden Fall geholfen. Und dann auch natürlich irgendwie Reflexionen dann innerhalb der gesamten Gruppe. Wenn dann nochmal andere Meinungen und Blickwinkel, die dazu kamen. Das (...) hat aufjeden Fall geholfen, da halt irgend- 
wie mit sich selbst auch irgendwie mal zu anderen Schlüssen vielleicht einfach zu kommen. Ja" (Boris)

Dazu kommen die formellen, durch die Exkursionsleitung initiierten Reflexionen. Ziel solcher Reflexionen sollte es nicht ausschließlich sein, erworbenes Faktenwissen zu festigen, wie z. B. die Funktionsweise des Wassertanks o. ä. Vielmehr sollte es ein Ziel sein, aufzuschlüsseln, was wieso für wen aus welcher Perspektive irritierend war, ,fremd' erschien, wieso und mit welchen Konsequenzen. Es geht also nicht (nur) darum, ,(mehr) Wissen' über „Fremde' zu erlangen, um sie besser zu verstehen: „Denn solches, Wissen kann, wenn es nicht reflexiv ist, das Sehen und Analysieren einer konkreten Situation sogar verhindern." (Kalpaka 2015, 309 f.). Ziel ist es vielmehr, sich immer wieder bezüglich des eigenen, Wissens' verunsichern zu lassen, also sich die vermeintlich gültigen inkorporierten Wissensbestände über andere Gruppen bewusst zu machen und diese zu hinterfragen" (Schröder 2016, 22; Hervorh. i. Orig.). Irritationen durch Fremdheitserfahrungen reflexiv aufzuarbeiten und neu zu verstehen, fassen wir in drei Punkten zusammen:

Punkt 1: Was hat wie individuell irritiert, bestehende Vorstellungen gebrochen? Was wurde wie als etwas ,Fremdes' erspürt, erlebt und als solches gedeutet?

Punkt 2: Wie wurde mit dem irritierenden ,Fremden` in der konkreten Situation umgegangen? Welche Haltungen und Bereitschaften zur Öffnung für Neues oder Strategien zum Ausweichen und Beharren auf Altem können rückblickend entdeckt werden?

Punkt 3: Wie kann das Geworden-Sein dieser Empfindung und Deutung von ,Fremdheit' erklärt werden?

Insbesondere hinsichtlich des dritten Punkts bedarf es einer näheren Betrachtung:

\section{,Fremdheit' als Konstrukt gesellschaftlicher Diskurse}

,Fremdheit' ist phänomenologisch gesehen das, was die Exkursionsteilnehmenden in der Begegnung mit Ausschnitten der Welt leiblich spüren, weil es sie irgendwie innerlich berührt, irritiert, fraglich macht. Bedeutsam ist dabei jedoch auch, dass, Fremdheit in starkem Maße gesellschaftlich konstruiert wird: Menschen anderer Hautfarben etwa werden als , die Fremden' kommuniziert. Derartige Klassifizierungen bestimmter Bevölkerungsgruppen fungieren als Bedeutungsträger innerhalb von Diskursen, die Differenzen herstellen. Dies zeigt nicht nur ein Blick in die Geschichte Afrikas, sondern auch in die ganz aktuellen Diskurse um Flüchtlingsbewegungen und Grenz- ziehungen. ,Fremdheit' ist dann nicht mehr lediglich das subjektive Erleben von Etwas, das sich der subjektiven Ordnung entzieht (vgl. Waldenfels 1997) und neugierig macht, sondern verortet sich innerhalb eines Diskurses um Klassifizierungen, die dazu dienen, die Bevölkerung einzuteilen, wobei Fragen von Macht eine ideologische Instanz konstituieren. In diesem Sinne muss der Rassismus als ideologischer Diskurs und damit als soziale Praxis erwähnt werden: Rassen existieren nicht als solche, „aber Rassismus kann in sozialen Praxen produziert werden" (Hall 2000, 7). Seine Funktion ist es, mit der Konstruktion ,des Anderen' eigene Identifikationen abzusichern und die Welt in jene einzuteilen, die dazugehören und in jene, die nicht dazugehören. Damit werden privilegierende Unterschiede markiert, das Fremde an einem fernen Platz fixiert, vom eigenen Selbst in sicherer Entfernung gehalten (vgl. ebd., 15; Rommelspacher 2009, $25 \mathrm{ff}$.). Dies gilt für Ostafrika in ähnlicher Weise in Bezug auf die vermeintliche Einteilung der Bevölkerung in ,Stämme': „Die Vorstellung, die afrikanische Bevölkerung bestehe aus wenig differenzierten, kleinen und voneinander klar abgrenzbaren Gesellschaften oder Gemeinschaften - oder gar ,Stämmen ' - ist ein Mythos, das Resultat eines historisch gewachsenen Afrikabildes zur Legitimierung von Ungleichheit und Unterdrückung“" (Krenceyová 2017, 191). Zusammengefasst: Vorstellungen von Fremdheit können ihren Ursprung auch in gesellschaftlich geschaffenen Diskursen haben - und damit auch ein Empfinden von Fremdheit bedingen. Um im didaktischen Interesse einer Erfahrungsbildung auf Exkursionen die Irritationen durch ,Fremdes' reflexiv aufzuarbeiten, ist folglich das Geworden-Sein dieser Empfindungen und Deutungen von ,Fremdheit' (Punkt 3) mit den Teilnehmer/innen in den analytischen Blick zu nehmen. Es gilt zu erkennen, ob es sich um individuelles, leiblich gespürtes Getroffensein handelt und die gestiftete innere ,Unruhe' (Waldenfels 1997, 42) zum produktiven Umdenken, Umdeuten, Auseinandersetzen und Neu-Verstehen auffordert (Punkt 2). Und es gilt zu ergründen, inwiefern Spüren, sowie Hören über ,Fremdheit' (Punkt 1) ein gesellschaftlich bedingtes und politisches Produkt ist. Biographische und kulturelle Prägung beeinflussen, welche Ordnungen in ,Unruhe' geraten. Es gilt daher zu hinterfragen, inwiefern ,Fremdheits-Begegnungen' Konsequenzen der gesellschaftlich geprägten Sozialisation sind und die Interpretation von ,Fremde "und ,Unruhe dementsprechend gelernt wurde und mit einer xenophoben Abwehrhaltung verknüpft wird, ohne die Eigenheit des Eigenen einer Befragung zu unterziehen. Dadurch kann eine Sensibilität erwachsen, die die angehenden Lehrkräfte zu einem machtsensiblen Umgang mit Ansätzen interkulturellen Lernens befähigt (vgl. 
Schröder 2016). Die soziale Konstellation der Reisegruppe ,Exkursion' bietet dabei einen Schatz an unterschiedlichen Empfindungen und Interpretationen von ,Fremdheit'. Wird dies aus der Metaperspektive betrachtet und die Frage nach dem Geworden-Sein dieser Mehrperspektivität und Mehrdimensionalität gestellt, so liegt hierin zugleich ein Schatz, um die Konstruiertheit von Raumbildern aufzudecken: „Wir stellen selbst neues Wissen her, wir produzieren Raum, indem wir unterschiedlichste Aspekte, den mentalen, sozialen und materiellen Raum in einen Zusammenhang bringen, der nicht nur durch Harmonie, sondern auch durch Widersprüche gekennzeichnet ist. Und diese Widersprüche lassen sich im Mikrokosmos Exkursion nutzen, um eigene Fragen zu stellen und an der Grenze unserer eigenen Bedeutungen und Verstehensbemühungen nachzuforschen und neue Antworten zu finden" (Dickel \& Scharvogel 2013, 183). Wie dies gelingen kann, wurde in den Kapiteln 3.1. und 3.2 ausgeführt.

\section{Fazit: Zum Potenzial von Exkursionen in der Ausbildung von Lehrkräften}

„Wer [...] ins Feld geht, lernt eher unbeabsichtigt genau so viel über sich selbst und die eigene Kultur wie über die Menschen, die er studiert. Die Situationen, die einen das Vertraute in neuem Licht erblicken lassen, scheinen, während man sie erlebt, gewöhnlich keine größere Bedeutung zu haben. Aber sie summieren sich. Je mehr man sich in ihrer Kultur wohl fühlt, desto bizarrer beginnt einem die eigene anzumuten" (Bohannan \& van der Elst 2003, 72; Hervorh. i. Orig.). Exkursionen sind in besonderem Maße auch Ort derartiger Selbstbildung, denn - wie die Beispiele gezeigt haben - ereignen sich Fremdheitsbegegnungen gerade außerhalb pädagogischer Inszenierungen. So war der Festakt an der Ndauni Primary School ebenso unerwartet wie die irritierende Begegnung mit dem Schulleiter der Primary School in Ol Donyo Sabuk. Die entsprechenden Situationen entziehen sich also jeglicher Vorbereitung: Sie wurden weder vom Dozenten geplant oder inszeniert, noch konnten sich die Studierenden, etwa im Rahmen des Vorbereitungsseminars zur Exkursion, konkret darauf einstellen und mögliche Reaktionen oder Verhaltensweisen vorbereiten. Auch wenn derartige Bildungsanlässe also bisweilen unbeabsichtigt entstehen, so muss doch gerade mit einer bewussten Reflexion darauf reagiert werden. Böing/Sachs $(2007,40)$ weisen als einer der zentralen Charakteristika für fachdidaktische Exkursionen eine „analytisch-reflexive Nachbereitungsphase“ aus. Einer gründlichen Reflexion, alleine und mit der Exkursionsgruppe, hinreichend Zeit zu geben, erscheint als elementar für die Wirkung von Exkursionen. Insofern erscheint neben der Frage „Was sollen Schülerinnen und Schüler über Afrika lernen?" (Schrüfer 2013) folgende Frage für die erste Phase der Lehrer/innenbildung von Relevanz: Was sollen angehende Lehrkräfte über ,Afrika' lernen, wie sollen sie Mensch und Raum begegnen, um etwas über ,Afrika' und sich selbst in Erfahrung zu bringen, also ein Bewusstsein zu entwickeln, um darauf aufbauend ausgewählte Aspekte Subsahara-Afrikas in angemessener Weise im Schulunterricht thematisieren zu können?

Dieser Beitrag hat versucht, einige Facetten einer möglichen Antwort auf diese Frage aufzuzeigen. Es wurde deutlich, dass eine Methode, die sich an der pädagogischen Irritation orientiert und das Fremde als dasjenige aufgreift, das jetzt - zuvor Selbstverständliches - seiner Verständlichkeit beraubt ist und Neuordnungen provoziert, wirkmächtig sein kann. Diese bewusste Form reflexiver Raumwahrnehmungen kann auch als „Selbstreflexive Geographien“ (Eberth 2016) bezeichnet werden, da es nicht bei der Betrachtung äußerer Aspekte verbleibt, sondern ein Prozess der Selbsterkenntnis angebahnt wird.

Die Fremdheitsbegegnung vor Ort offenbart sich also in höchstem Maße gewinnbringend, da sie zur Veränderung von Vorstellungen, Einstellungen und Haltungen zukünftiger Multiplikatoren zu ,Afrika beiträgt (vgl. auch Wilhelmi 2015, 47). „To become a professional, one must learn not only to think in certain ways but also to perform particular skills, and to practice or act in ways consistant with the norms, values, and conventions of the profession" (Shulman 2002, zit. in Meyer 2011, 188). Theoretisches Wissen über inter- und transkulturelles Lernen reicht daher nicht aus, um als Lehrkraft den Rahmen für einen authentischen Unterricht zu ermöglichen. Es bedarf der leiblichen Begegnung im zunächst als fremd verstandenen Raum, um ein gewisses $\mathrm{Ma}$ an Transkulturalität zu leben.

Die hier vorgestellten exemplarischen Beispiele zeigen, dass gerade Exkursionen die richtige methodische Großform darstellen können, um derartige Stimuli zu provozieren und somit durch die Konfrontation mit etwas, das den vorher fraglosen Gegenstand oder Umstand ,wie eine Ohrfeige' in ein Problem verwandelt, bestehende Selbst- und Raumbilder neu zu figurieren. Darin liegt Potenzial, dass „der Einzelne immer besser in die Lage kommt, selbstbestimmt zu leben und die eigenen Entscheidungen zu verantworten. Freie Entscheidungen auch angesichts der Ungewissheit und Fragilität des Alltags sind nicht außerhalb des gesellschaftlichen Systems zu finden, sondern nur in kritischer Auseinandersetzung mit der gesellschaftlichen Ordnung" (Dickel 2016, 54). Insofern leistet der aufgezeigte Ansatz auch einen klaren Bezug zur politischen Bildung, da Aspekte globaler Disparitäten implizit Be- 
rücksichtigung finden, aber auch die eigene Rolle und das Eingebundensein in globale Strukturen reflektiert werden. Politisch-geographische Analyseperspektiven in die Ausbildung von Lehrerinnen und Lehrer zu integrieren, wird ausdrücklich gefordert (vgl. Reuber 2016, 117). Der auf verschiedenen Ebenen deutlich werdende Wirkungsgrad der Exkursion wiederum legitimiert den mit einer derartigen Reise einhergehenden immensen $\mathrm{CO}_{2}$-Ausstoß, der aus der Perspektive einer kritischen Umweltbildung kritisiert werden könnte, der aber durch Kompensationsmaßnahmen wie dem gemeinsamen Pflanzen von Bäumen im Kakamega-Forest zumindest ansatzweise ausgeglichen bzw. zum Bestandteil von Reflexionsprozessen wurde.

Die Begegnungen mit Fremden im Unterwegssein können zum einen als dasjenige gedeutet werden, das Erfahrungsprozesse anstößt. Das Fremde zeigt sich so als Potenzial, bisher unbemerkte, unhinterfragte Selbstverständlichkeiten zu verunsichern und Exkursionsteilnehmer/innen dazu anzustoßen, etablierte Selbst- und Weltbilder zu überdenken, ggf. zu verwerfen und umzudeuten. Zugleich besteht die Gefahr, dass einer solchen bildungsrelevanten Auseinandersetzung mit dem Fremden xenophob ausgewichen wird, das Fremde als etwas Feindliches aufgefasst und zur unterscheidenden Abgrenzung vom Eigenen genutzt wird.

Eine engagierte geographische Bildung verlangt verantwortlich zu ergründen, was Exkursionsteilnehmer/innen inwiefern als ,fremd' empfinden lässt: Die betroffen und neugierig machende Konfrontation mit etwas, dass sich dem bisher Vertrauten entzieht, und/oder das durch gesellschaftliche, mitunter rassistische Diskurse produzierte ,Fremde', dass wertende Klassifizierungen konstruiert und manifestiert (vgl. Hall 1990; Rommelspacher 2009, 25 f.). Für die Exkursionsdidaktik bedeutet das: $\mathrm{Ob}$ die irritierenden Konfrontationen mit Fremdem über ein xenophobes Ausweichen, über ein singuläres Erlebnis, über ein Verhaften in Gewohntem hinaus zu bildungsrelevanten Erfahrungen werden, hängt von ihrer Weiterführung ab. Der reflexiven Aufarbeitung dessen, was subjektiv betroffen macht, wie damit umgegangen wird und was diese Gefühle und Praktiken auslöst, kommt damit ein entscheidender Stellenwert zu. Hierin liegt eine Chance, subjektives Verstehen des ,Eigenen' und ,Fremden' mit gesellschaftlichen Prägungen ins Verhältnis zu setzen und reflexiv neu zu verstehen.

\section{Literatur}

Abraham, A. (2002): Der Körper im biographischen Kontext. Wiesbaden: VS.

Böing, M. \& U. Sachs (2007): Fachdidaktische Exkursionen. Ein Baustein zur Förderung exkursionsdidaktischer
Kompetenzen in der Lehrerbildung. In: Geographie und Schule 169, 39-46.

Bohannan, P. \& D. van der Elst (2003): Fast nichts Menschliches ist mir fremd. Wie wir von anderen Kulturen lernen können. Wuppertal: Peter Hammer.

Buck, G. (1989): Lernen und Erfahrung - Epagogik. Zum Begriff der didaktischen Induktion. 3. Auflage. Darmstadt: Wissenschaftliche Buchgesellschaft.

Dickel, M. (2006): TatOrte - Zur Implementation neuer Raumkonzepte im Geographieunterricht. In: Dickel, M. \& D. Kanwischer (Hrsg.): TatOrte. Neue Raumkonzepte geographiedidakatisch inszeniert. Berlin/Münster: LIT, 7-20.

Dickel, M. (2016): Der Sprung ins Denken. Geographie als Politische Bildung. In: Budke, A. \& M. Kuckuck (Hrsg.): Politische Bildung im Geographieunterricht. Stuttgart: Franz Steiner. 47-55.

Dickel, M. \& M. Scharvogel (2013): Geographische Exkursionspraxis: Erleben als Erkenntnisquelle. In: Kanwischer, D. (Hrsg.): Geographiedidaktik. Ein Arbeitsbuch zur Gestaltung des Geographieunterrichts (= Studienbücher der Geographie), Stuttgart: Bornträger, 176-185.

Dickel, M. \& A. Schneider (2013): Über Spuren. Geographie im Dialog. In: Zeitschrift für Didaktik der Gesellschaftswissenschaften 1, 56-75.

Dörpinghaus, A. (2009): Sorge um Bildung - vom lebenslangen Sterben. In: Ricken, N., H. Röhr, J. Ruhloff \& K. Schaller (Hrsg.): Umlernen. Paderborn: Wilhelm Fink, 35-46.

Eberth, A. (2016): Partizipation junger Zivilgesellschaften in den Slums von Nairobi - ein Beitrag zur Politischen Bildung im Geographieunterricht. In: Budke, A. \& M. Kuckuck (Hrsg.): Politische Bildung im Geographieunterricht. Stuttgart: Franz Steiner, 211-219.

Falk, G. C. (2015): Exkursionen. In: Reinfried, S. \& H. Haubrich (Hrsg.): Geographie unterrichten lernen. Die Didaktik der Geographie. Berlin: Cornelsen, 150-153.

Fromme, J. (2001): Irritation als ein zentrales Motiv für Lernen und Bildung. In: Vierteljahreszeitschrift für wissenschaftliche Pädagogik 77, 409-428.

Gebauer, G. \& Wulf, C. (2003): Mimetische Weltzugänge. Soziales Handeln - Rituale und Spiele - ästhetische Produktionen. Bonn.

Glasze, G. \& A. Mattissek (2009): Diskursforschung in der Humangeographie. Konzeptionelle Grundlagen und empirische Operationalisierungen. In: Glasze, G. \& A. Mattissek (Hrsg.): Handbuch Diskurs und Raum. Bielefeld: Transcript, 11-59.

Hall, S. (2000): Rassismus als ideologischer Diskurs. Vortrag, gehalten am 17.05.1989 in Hamburg. In: Rätzel, N. (Hrsg.): Theorien über Rassismus. Hamburg: Argument.

Horton, J. \& P. Kraftl (2014): Cultural Geographies. An Introduction. Abingdon/New York: Routledge.

Just, K. G. (1966): Übergänge. Problem und Gestalten der Literatur. Bern: Francke.

Kalpaka, A. (2015): Pädagogische Professionalität in der Kulturalisierungsfalle - Über den Umgang mit ,Kultur 
in Verhältnissen von Differenz und Dominanz. In: Leiprecht, R. \& A. Steinbach (Hrsg.): Schule in der Migrationsgesellschaft. Ein Handbuch: Bd. 2 Sprache - Rassismus - Professionalität. Schwalbach: Debus Pädagogik, 289-312.

Kersting, P. (2011): AfrikaSpiegelBilder und Wahrnehmungsfilter: Was erzählen europäische Afrikabilder über Europa? In: Kersting, P. \& K. W. Hoffmann (Hrsg.): AfrikaSpiegelBilder. Reflexionen europäischer Afrikabilder in Wissenschaft, Schule und Alltag (= Mainzer Kontaktstudium Geographie 12), Mainz: Geographisches Institut der Johannes Gutenberg-Universität, 3-10.

Koller, H.-C. (2012): Fremdheitserfahrungen als Herausforderung transformatorischer Bildungsprozesse. In: Bartmann, S. \& O. Immel (Hrsg.): Das Vertraute und das Fremde. Differenzerfahrungen und Fremdverstehen im Interkulturalitätsdiskurs. Bielefeld: Transcript, 157-176.

Kokemohr, R. (2007): Bildung als Welt- und Selbstentwurf im Anspruch des Fremden. Eine theoretisch-empirische Annäherung an eine Bildungstheorie. In: Koller, H.-C., W. Marotzki \& O. Sanders (Hrsg.): Bildungsprozesse und Fremdheitserfahrung. Bielefeld: Transcript, 13-68.

Knox, P. \& S. Marston (2016): Human Geography. Places and Regions in Global Context. Seventh Edition. Harlow: Pearson.

Krenceyová, M. (2017): Reflexionen zu Kultur und Gesellschaft. In: Eberth, A. \& A. Kaiser (Hrsg.): Ostafrika. Geographie, Geschichte, Wirtschaft, Politik. (WBGLänderkunden). Darmstadt: Wissenschaftliche Buchgesellschaft, 191-195.

Lossau, J. (2012): Postkoloniale Impulse für die deutschsprachige Geographische Entwicklungsforschung. In: Geographica Helvetica 67, 125-132.

Meyer, C. (2011): Professionelle Kompetenz von Geographielehrkräften. Ansätze für empirische Forschung. In: Meyer, C., R. Henrÿ, R. \& G. Stöber (Hrsg.): Geographische Bildung. Kompetenzen in didaktischer Forschung und Schulpraxis (= Geographiedidaktische Forschungen 47), Braunschweig: Westermann, 184-201.

Meyer-Drawe, K. (2008): Diskurse des Lernens. München: Wilhelm Fink.

Mönter, L. (2013): Interkulturelles Lernen. In: Rolfes, M. \& A. Uhlenwinkel (Hrsg.): Metzler Handbuch 2.0 Geographieunterricht. Ein Leitfaden für Praxis und Ausbildung. Braunschweig: Westermann, 87-95.

Reinfried, S. (2015a): Vorurteile und Stereotype bewusst machen. In: Reinfried, S. \& H. Haubrich (Hrsg.): Geographie unterrichten lernen. Die Didaktik der Geographie. Berlin: Cornelsen, 74-79.

Reinfried, S. (2015b): Schülervorstellungen bei der Gestaltung von Unterricht berücksichtigen. In: Reinfried, S. \& H. Haubrich (Hrsg.): Geographie unterrichten lernen. Die Didaktik der Geographie. Berlin: Cornelsen, 80-81.

Reuber, P. (2016): Politische Geographie im Erdkundeunterricht - Möglichkeiten und Perspektiven. In: Budke, A. \& M. Kuckuck (Hrsg.): Politische Bildung im Geographieunterricht. Stuttgart: Franz Steiner, 109-117.
Rhode-Jüchtern,T. \&A. Schneider(2012): Wissen, Problemorientierung, Themenfindung im Geographieunterricht (= Kleine Reihe - Geographie), Schwalbach: Wochenschau.

Rommerlspacher, B. (2009): Was ist eigentlich Rassismus? In: Melter, C. \& P. Mecheril (Hrsg.): Rassismuskritik. Bd. 1. Schwalbach: Wochenschau.

Schirp, J. (2008): Abenteuer - Ein Weg zur Jugend? Das Fremde als Schlüsselthema in der Abenteuer- und Erlebnispädagogik. Marburg: Barbara Budrich.

Schlottmann, A., A. Mösgen \& T. Böhm (2014): Räumliche Sozialisation und Schule - Theorie und Praxis eines Bausteins humangeographischer Lehrerbildung. In: Zeitschrift für Geographiedidaktik 42 (2), 97-113.

Schröder, B. (2016): Machtsensible geographiedidaktische Konzepte des interkulturellen Lernens - Potenziale einer postkolonialen Perspektive. In: GW-Unterricht 144 (4), 15-28.

Schrüfer, G. (2013): Was sollen Schülerinnen und Schüler über Afrika lernen? In: Praxis Geographie 43 (7-8), 8-10.

Schrüfer, G. \& G. Obermaier (2014): Wahrnehmung von Räumen. Konstruktion und Bewertung aus unterschiedlichen Perspektiven. In: Schwarz, I. \& G. Schrüfer (Hrsg.): Vielfältige Geographien. Entwicklungslinien für Globales Lernen, Interkulturelles Lernen und Wertediskurse (= Erziehungswissenschaft und Weltgesellschaft 7), Münster: Waxman, 171-186.

Sefa Dei, G. J. (2012): Teaching Africa. Towards a Transgressive Pedagagoy (= Explorations of Educational Purpose 9), Heidelberg/London: Springer.

Segbers, T. \& D. Kanwischer (2015): Ethnographische Feldforschung im Kontext der Reise - Teilnehmende Beobachtung und Tagebuchanalyse als erkenntnisleitende Forschungsinstrumente. In: Budke, A. \& M. Kuckuck, M. (Hrsg.): Geographiedidaktische Forschungsmethoden (= Praxis Neue Kulturgeographie 10), Münster: LIT, 295-317.

Stein, C. (2013): Schulen sorgen für Wasser in Ostafrika. Wie motiviert man Schüler für Eine-Welt-Themen? In: Praxis Geographie 43 (7-8), 24-29.

Tengelyi, L. (2002): Vom Erlebnis zur Erfahrung. Phänomenologie im Umbruch. In: Hogrebe, W. in Verbindung mit J. Bromand (Hrsg.): Grenzen und Grenzüberschreitungen. XIX. Dt. Kongress für Philosophie. Berlin: Akademie, 788-800.

Waldenfels, B. (1997): Topographie des Fremden. Studien zur Phänomenologie des Fremden 1. Frankfurt am Main: Suhrkamp.

Waldenfels, B. (1999): Der Stachel des Fremden. Frankfurt am Main: Suhrkamp.

Wilhelmi, V. (2015): Warum Reisen nach Ruanda wichtig sind. In: Praxis Geographie 45 (4), 48-50. 